\title{
UN MODELO TRANSACCIONAL O CONTEXTUALIZADO DE AUTONOMÍA EN PERSONAS CON DEMENCIA
}

\author{
A TRANSACTIONAL OR CONTEXTUALIZED MODEL \\ OF AUTONOMY IN PERSONS WITH DEMENTIA
}

\section{Jean Gajardo J. ${ }^{\text {, Diego Cifuentes L. }}{ }^{2}$}

\begin{abstract}
Resumen
La demencia es una condición que se caracteriza por la alteración de la memoria y otras funciones cognitivas y de la regulación conductual, de carácter adquirido y que repercute el desempeño y la funcionalidad de la persona. La demencia ha sido declarada una prioridad para la salud pública debido a su creciente frecuencia y es una de las principales causas de discapacidad y dependencia en las personas adultas mayores. La alteración cognitiva presente en la demencia se relaciona con la autonomía de las personas, lo que representa desafíos particulares para la Terapia Ocupacional como disciplina que busca la promoción de participación y de autonomía en las personas. Por medio de este ensayo se realiza una discusión en torno a diferentes paradigmas sobre la autonomía en la demencia, para visualizar el desarrollo de un marco conceptual de dicha relación que expanda su comprensión desde la determinación individual hacia la relación con los demás, el ambiente, la agencia y el transaccionalismo, permitiendo vislumbrar un mayor potencial de intervención de Terapia Ocupacional y de estudio desde la ocupación.
\end{abstract}

\section{Palabras clave:}

Demencia, autonomía, perspectiva transaccional, ambiente, agencia, ocupación

\begin{abstract}
Dementia is a condition that features the impairment of cognition and other cognitive and behavioral functions. Dementia is an acquired condition that impacts performance and functionality and it has been declared a public health priority due to its increasing frequency and because it represents one of the major causes of disability and dependence among older adults. The cognitive impairment that dementia implies has an impact on the person's capability to be autonomous, which is at the same time an important challenge to address by occupational therapy as a profession and discipline that aims to promote participation and autonomy. This essay offers a discussion about paradigms and notions about autonomy in dementia, in order to develop a conceptual framework that enhances an alternative notion of autonomy in dementia better related to the environment, agency and a transactional perspective of occupation.
\end{abstract}

\section{Key words:}

Dementia, autonomy, transactional perspective, agency, environment, occupation

1 Terapeuta Ocupacional, Licenciado en Ciencias de la Ocupación Humana, Máster en Gerontología Social. Doctorante en Salud Pública, Universidad de Chile. Profesor Asistente en Docencia, Escuela de Terapia Ocupacional. Facultad de Medicina. Universidad de Chile. Becario nacional Conicyt. Independencia 1027. Tel 9786342. jgajardo@med.uchile.cl

2 Terapeuta Ocupacional, Licenciado en Ciencias de la Ocupación Humana, Diplomado en el Modelo de Ocupación Humana, Estudiante Máster Europeo en Terapia Ocupacional. Académico Escuela de Terapia Ocupacional. Facultad de Medicina. Universidad de Chile. Independencia 1027. Tel 9786589. dcifuentes@med.uchile.cl 


\section{INTRODUCCIÓN}

En el año 2002, las personas de 60 años y más representaban el $12,95 \%$ de la población total en Chile. Se ha proyectado que esta cifra aumentará a un $17,29 \%$ en el año 2020, y alcanzará el 28\% en el año 2050 (Instituto Nacional de Estadísticas, 2008). En dicho contexto, se ha estimado también el aumento de los grupos de edades más avanzadas en la población de 60 años y más, siendo de este modo el grupo de 80 años y más es el que aumentará de forma más significativa según las proyecciones demográficas de nuestro país (Instituto Nacional de Estadísticas, 2004).

Una de las principales consecuencias del envejecimiento poblacional es la transición epidemiológica que determina un cambio en el perfil de salud - enfermedad de los países. De este modo, de la mano del envejecimiento aumentan su frecuencia las condiciones de salud de carácter crónicas y no transmisibles, tales como la demencia (Szot, 2003). Definida desde un paradigma biomédico, la demencia es un síndrome clínico de deterioro cognitivo adquirido y progresivo, cuyos pilares fundamentales son los déficits cognitivos, los problemas conductuales y el impacto relevante en la funcionalidad o capacidad de llevar a cabo las actividades diarias (Fuentes \& Slachevsky, 2005). El término "demencia" es usualmente utilizado para referir dicho síndrome de naturaleza mayormente cognitiva y progresiva, que es causado por otras enfermedades, dentro de las cuales se encuentra la enfermedad de Alzheimer, siendo ésta la principal causa de demencia en personas de 60 años o más, por lo que en su nominación suele utilizarse indistintamente los términos demencia o demencia tipo Alzheimer (Donoso, 2003).

En Chile, a partir de datos de prevalencias y proyecciones internacionales, se estima que cerca de 200 mil personas presentarían demencia en la actualidad y en coherencia con el contexto global, se proyecta la triplicación de esta prevalencia hacia el año 2050 (Corporación Profesional Alzheimer y otras demencias, 2012; Quiroga, Albala, \& Klaasen, 2004).
La demencia es una condición de salud con impactos variados que incluye al individuo y su entorno familiar y social, y representa una de las principales causales de discapacidad en las personas adultas mayores, siendo en Chile la tercera causa específica de pérdida de Años de Vida Saludables (AVISA) en este grupo etario (Departamento de Salud Pública Pontificia Universidad Católica de Chile, 2008).

Debido a la naturaleza de sus síntomas, la relación entre la demencia y la autonomía es un tema de frecuente debate tanto en términos clínicos como bioéticos. La alteración cognitiva presente en la demencia, frecuentemente de carácter progresiva, genera con frecuencia la asunción de una relación directa entre la incapacidad de autonomía y el nivel cognitivo (Garand, Lingler, Conner, \& Dew, 2009; Organización Mundial de la Salud, 2012). Esta asunción a priori puede Ilevar a la limitación prematura de autonomía y dignidad de las personas con demencia, lo que como consecuencia favorece la pérdida de autoestima asociada con dicho estigma (Robinson et al., 2008).

¿Cómo puede comprenderse la autonomía y qué particularidades presenta en la demencia? ¿Qué racionalidades para dicha relación tienen mayor pertinencia para el quehacer de la Terapia Ocupacional y el estudio de la ocupación en las personas con demencia? ¿Qué elementos son posibles de visualizar hacia la construcción de un marco de comprensión de la autonomía en la demencia que enfatice en la ocupación y la participación?

A través de una revisión bibliográfica y análisis temático, este ensayo busca responder a las preguntas previamente señaladas con el propósito de aportar con elementos que permitan la generación de la estructura inicial de un modelo de autonomía en demencia basado en perspectivas de la ocupación, transaccionalismo y agencia.

\section{Paradigmas en la demencia}

La palabra demencia proviene del latín, y sus componentes léxicos son el prefijo de, el cual alude a alejamiento o privación; mens o mentis, que 
alude a la mente; y finalmente el sufijo cia, que alude a cualidad (Diccionario Etimológico, 2014). Luego, el término demencia refiere entonces a un sujeto que es privado o alejado de su mente.

La definición de demencia usada con mayor frecuencia responde a la interpretación de sus manifestaciones clínicas, según los tres clasificadores de mayor relevancia en la clínica actual: DSM-IV V, CIE-10 y NINCS-ARDRA (National Institute of Neurologic, Communicative Disorders and Stroke - Alzheimer's Disease and Related Disorders Association). De manera global, dichos clasificadores enfatizan en las alteraciones cognitivas (memoria y otras funciones) y el impacto que tienen éstas en la ejecución de actividades ocupacionales y/o sociales en comparación con las capacidades previas.

Si bien la noción de demencia ha emergido principalmente como término generado desde la biomedicina en pos de consensuar criterios para su diagnóstico y caracterización, el análisis de la demencia como concepto y sus significados, ha progresivamente adquirido importancia como un factor reconocido que influye en la actitud de la persona misma y su entorno social en relación con su diagnóstico y por ende en la experiencia de enfermedad (Pesonen, Remes, \& Isola, 2013; Wadley \& Haley, 2001). Estos significados y percepciones son elementos importantes al analizar la relación demencia - autonomía.

De acuerdo a Cuijspers y van Lente (2014) la discusión en torno al concepto de demencia y enfermedad de Alzheimer se ha generado históricamente con tensiones entre modelos biomédicos y psicosociales en su comprensión y consideración de necesidades. De este modo, es posible identificar distintas formas de aproximación a la definición de la demencia y la enfermedad de Alzheimer. Se ha propuesto que los principales modelos teóricos descritos en la actualidad son el modelo biomédico, modelo psicosocial, modelo de gerontología y un modelo integrado (Innes \& Manthorpe, 2013), cuyas características son descritas en la Tabla 1. Cada una de estas aproximaciones enfatiza nociones particulares del problema, y sus consecuencias y necesidades vinculadas. 
Tabla 1. Características de diferentes paradigmas en torno a la definición de la demencia

\begin{tabular}{|c|c|}
\hline Paradigma & Características \\
\hline Biomédico & $\begin{array}{l}\text { La demencia se comprende como una enfermedad con síntomas a tratar. } \\
\text { Las políticas públicas deben proporcionar marcos de acción terapéutica. } \\
\text { La investigación en demencia se enfocará en probar el impacto de intervenciones para los } \\
\text { síntomas propios de la demencia. } \\
\text { Las prácticas clínicas se enfocarán en aliviar síntomas de la enfermedad. }\end{array}$ \\
\hline Psicosocial & $\begin{array}{l}\text { La demencia se visualiza a un nivel individual como una experiencia única vivida por la } \\
\text { persona. } \\
\text { La política pública requiere promover y lograr aproximaciones individualizadas al cuidado } \\
\text { y tratamiento. } \\
\text { La investigación se enfocará en el desarrollo de tratamiento, cura, y prevención, tanto para } \\
\text { la persona con demencia como su cuidador/a. } \\
\text { Las prácticas clínicas se enfocarán en trabajar con los individuos para lograr acciones } \\
\text { moldeadas según las necesidades y preferencias de la persona con demencia. }\end{array}$ \\
\hline Gerontológico & $\begin{array}{l}\text { La demencia se visualiza como una experiencia y discapacidad situada en un contexto } \\
\text { social, ambiental y estructural particular. } \\
\text { La política pública requiere comprender y reflexionar sobre la posición social que las } \\
\text { personas con demencia ocupan. } \\
\text { La investigación en demencia se enfocará en contextualizar las experiencias de vida de } \\
\text { personas con demencia en sus contextos sociales, políticos y económicos particulares. } \\
\text { Las prácticas clínicas debiesen promover desarrollos basados en las visiones de los } \\
\text { usuarios, una aproximación basada en derechos, e imágenes positivas de la vejez. }\end{array}$ \\
\hline Integrado & $\begin{array}{l}\text { La demencia es una condición que necesita ser comprendida desde una perspectiva } \\
\text { biomédica, psicosocial y gerontológica. } \\
\text { La política pública necesita reflejar conocimiento biomédico, preocupaciones psicosociales } \\
\text { sobre el individuo al mismo tiempo que reconocer las desventajas que enfrentan los adulto } \\
\text { mayores con discapacidad. } \\
\text { La investigación en demencia puede enfocarse en niveles micro y macro para promover } \\
\text { una mejor comprensión de las experiencias y visiones de los profesionales, cuidadores y } \\
\text { las personas con demencia. Esto fomentaría el involucramiento en el debate político, en las } \\
\text { actitudes sociales y las creencias sobre la demencia y el cuidado de calidad. } \\
\text { Las prácticas clínicas necesitan considerar al individuo y su déficit neurológico de igual } \\
\text { modo que las limitantes estructurales para proveer cuidado de alta calidad. }\end{array}$ \\
\hline
\end{tabular}

Fuente: Innes, 2013. Traducción propia.

Por su parte, Zeilig (2013) reflexiona sobre la demencia como una condición biológica que se desarrolla en una historia personal y como una metáfora cultural considerando las comprensiones colectivas de la condición, que impregnan nuestras emociones hacia la demencia y la forma en la que interactuamos con las personas con esta condición. Entre sus principales conclusiones están el cómo la demencia y la enfermedad de Alzheimer se han constituido como términos similares en la comprensión colectiva. Del mismo modo, discute críticamente los discursos colectivos que relacionan la demencia y la vejez como condiciones de desastre y crisis, alimentados principalmente por los medios y la investigación científica biomédica. Zeilig refiere que analizar los guiones culturales sobre la demencia y la enfermedad de Alzheimer ayuda a revelar las asun- 
ciones subyacentes que impregnan las narrativas políticas, sociales y médicas que se generan en relación con estas condiciones. Lo que es más importante, la autora concluye que estas historias y estereotipos distancian a los sujetos sin demencia de aquéllos con demencia, y contribuyen a los sentimientos de aislamiento y el estigma que frecuentemente experimentan quienes presentan estas condiciones, especialmente en la etapa leve de la demencia.

Desde el poder de los discursos y significados en torno a la demencia se ha referido que ésta es un fenómeno fluido y muy dinámico y no es, como suele ser representado por los discursos médicos y sociales, una declinación irreversible y progresiva de la naturaleza humana del sujeto. Se propone que los esfuerzos debiesen considerar la complejidad de la demencia como una condición relacional y debiesen centrarse en la experiencia también dinámica de las personas que viven con la condición, enfatizando en la necesidad de transformar las actuales imágenes que existen sobre la demencia basadas en poderosos discursos fatalistas y destructivos, principalmente alimentados por la visión biomédica y los medios de comunicación, hacia discursos que posibiliten el respeto y una relación social de comprensión y creatividad que impregne los sistemas de cuidados que se institucionalicen (Mitchell, Dupuis, \& Kontos, 2013).

Como puede observarse, es posible distinguir diferentes aproximaciones a las implicancias de la demencia. Algunas de éstas enfatizan en el componente de capacidad individual, y otras en el componente relacional de la experiencia del sujeto con su entorno. Asimismo, puede notarse que algunas plantean una noción dual y estática de las capacidades (presente o ausente, suficiente o insuficiente, adecuado o inadecuado), mientras que otras establecen una mirada dinámica y con mayor situacionalidad de la experiencia de tener demencia. En este escenario, resulta pertinente preguntarse, ¿cuáles de estas consideraciones son pertinentes a la Terapia Ocupacional y la Ciencia de la Ocupación? ¿Cómo debiésemos observar el fenómeno de la demencia desde el quehacer profesional y disciplinar?

Las posturas actuales en torno al abordaje de la demencia adscriben mayor énfasis en los aspectos relacionales y dinámicos de ésta, con miras a sostener la idea que es posible vivir bien con la demencia (Organización Mundial de la Salud, 2012; Public Relations Institute of Australia Program Fight Dementia, 2011), postura que puede resultar contrastante a las visiones desde la tradición biomédica que han tendido a enfatizar la patología del fenómeno y la negación de las capacidades de decisión y autonomía en las personas con demencia desde un enfoque totalitarista, y por ende, limitando la posibilidad de acceso a participación y bienestar facilitado. Una reflexión local interesante de plantear es la realizada por Dörr (2005) al referir la noción de un sujeto demente como una realidad humana triste, carente de condiciones humanas esenciales como la autorreflexión y el manejo de sí mismo y el entorno, e incapaz de autocuidarse en ningún nivel.

Los énfasis que tienen las distintas miradas de la demencia representan marcos generales en los que se configuran las racionalidades sobre la autonomía en esta condición. De manera lógica, podría atribuirse al paradigma biomédico una noción de autonomía centrada en la capacidad individual y los factores neurocognitivos, y a los paradigmas con mayor integralidad y focalización en las experienciales, una racionalidad de la autonomía con mayor determinación desde el contexto del sujeto y las experiencias de oportunidad, poder y relación con su entorno. A continuación, tomando en consideración elementos teóricos sobre autonomía y demencia, se analizará distintas racionalidades que pueden establecerse para dicha relación.

\section{Racionalidades sobre la autonomía en la demencia}

Dekkers (2004) describe la autonomía a partir de su análisis etimológico. La palabra está compuesta por los términos autos y nomos, nociones que involucran la idea de un estado de guía propia, 
de determinación individual, proveniente desde el interior del sujeto sin interferencia externa. Dicha noción de la autonomía, eminentemente determinista, dual y basado en las condiciones internas propias del individuo ha sentado las bases para las discusiones teóricas en torno al concepto.

Desde esta perspectiva, resulta útil mencionar la propuesta conceptual que desarrolla Agich (1994), quien describe la existencia de dos miradas a la autonomía:

Autonomía estándar o abstracta, que comprende la asunción de un set de capacidades que el agente autónomo debe poseer para ser nominado como tal, incluyendo la capacidad de ser independiente, tomar decisiones racionales y conocer sus preferencias y deseos.

Autonomía real o de efecto, centrada en las características fenomenológicas o de los significados de la persona en su mundo real y actividades concretas. Esta aproximación rescata la historia personal, las creencias, los proyectos y valores que el sujeto pone en juego en su interacción con el mundo social.

El autor incorpora la noción de autonomía real o de efecto en respuesta a la noción de autonomía estándar, la cual propone como hegemónica en la comprensión del concepto. De este modo, la idea de autonomía se vuelve un problema más amplio derivando hacia las dificultades o características particulares de cada experiencia.

De manera complementaria y más reciente, $\mathrm{Ri}$ gaux (2011) analiza la construcción histórica del concepto de autonomía y en coherencia con lo propuesto por Agich, propone que las nociones de autonomía pueden ser clasificadas en dos polos: un polo doctrinario y un polo relacional. Para la autora el primero es el más frecuente y conceptualiza la autonomía como un producto de competencias internas propias del sujeto, tales como la memoria y el razonamiento. Bajo este prisma, las personas con demencia son fácilmente excluidas de ser consideradas como sujetos autónomos. Por su parte, el polo relacional propuesto por la autora enfatiza en las condiciones externas al sujeto como posibilitadoras de autonomía. Luego, los procesos, relaciones, instituciones, y políticas son determinantes en las oportunidades de actuar autónomamente en las personas con demencia. Desde este punto de vista, el polo relacional reconoce mayores posibilidades de inclusión a la persona con demencia ya que su autonomía no depende exclusivamente de sí misma sino que también en aquéllos que se relacionan con ella. La autora concluye en la necesidad de generar estrategias que aporten con la promoción de una concepción de autonomía amigable a la demencia.

A partir de lo planteado por Rigaux, emergen elementos iniciales de alusión a factores externos al sujeto como parte de la noción de autonomía, y al mismo tiempo, la naturaleza relacional y de interconexión que estos tienen.

Por otra parte, se ha sostenido que las personas con demencia son un grupo marginalizado que presenta alta vulnerabilidad de sufrir procesos de muerte social, comprendiendo éstos como aquéllos en los que las personas son consideradas no dignas de participación y por ende ser excluidas de circunstancias de vida particulares (como la investigación u otros procesos de búsqueda de bienestar institucionalizados) (Brannelly, 2011). Branelly problematiza los escenarios cotidianos de acción en los que las personas con demencia se ven excluidas debido a la naturalización de la incompetencia total, y también reconocer y analizar aquéllas que aporten en promover la participación de las personas con demencia. De este modo, la autora afirma que si los investigadores y profesionales vinculados a la demencia no son capaces de ver a una persona con demencia como socialmente viva y como potencial actor social, entonces los espacios de ejercicio de ciudadanía y personalidad no pueden ser facilitados, como se esperaría que fuese en el abordaje de la demencia. Desde esta mirada, emerge la noción de acción social y agencia, que será tratada luego en este manuscrito, como una consideración más hacia una visión de las personas con demencia.

Finalmente, desde una mirada neuropsicológica, una premisa esencial de considerar es que no obstante la indudable asociación que se produ- 
ce entre la demencia y la alteración cognitiva y conductual, el diagnóstico de demencia o enfermedad de Alzheimer no equivale de forma proporcional a incompetencia, debido a que dichos procesos neuropsicológicos son también relacionales y dependientes del ambiente y otros factores situacionales (Marson, 2001).

A partir de lo previamente descrito, es factible notar que la racionalidad de la autonomía en la demencia ha cambiado desde una perspectiva centrada en la capacidad individual y la valoración determinista, hacia una noción que enfatiza en la idea de factores múltiples, el dinamismo y la situacionalidad. Dicha conclusión representa un elemento relevante de considerar hacia el establecimiento de una aproximación de la Terapia Ocupacional a la autonomía en la demencia.

A continuación, se analiza algunos elementos de aporte en la construcción de un modelo de autonomía en la demencia a partir de las perspectivas de la ocupación, el transaccionalismo y la agencia. Dichas perspectivas corresponden a miradas teóricas sobre la naturaleza de la ocupación que resultan de aporte a la configuración de una racionalidad de la autonomía en la demencia que enfatice en aspectos relacionales y dinámicos y de mayor pertinencia a la Terapia Ocupacional.

\section{Elementos desde ocupación, agencia y transaccionalismo}

Tradicionalmente, una de las principales preocupaciones que ha tenido la Terapia Ocupacional se relaciona con la intervención y estudio de la ocupación a partir del desempeño observable, no obstante, las ocupaciones presentan complejidades inherentes y un significado situado para las personas que va más allá de dicho acto ejecutivo que usualmente denominamos desempeño (Hammell, 2004, 2009) y por ende, la ocupación no debiese comprenderse en términos únicamente categoriales (según adecuación o nivel de independencia o efectividad) sino que en términos de dimensiones de significado (Wilcock, 1998). A partir de lo anterior, si se reflexiona desde una mirada tradicional de la ocupación, una racionalidad de autonomía de polo externo o tradicional plantearía el énfasis categorial de "ser autónomo", mientras que una aproximación desde dimensiones de significado se centraría en el "hacer", "volverse", y "sentirse autónomo(a)".

Una comprensión situada de la ocupación es un marco de análisis también relevante hacia la generación de un modelo de autonomía en demencia, y de este modo, una perspectiva transaccional o contextualizada emerge como una alternativa posible para comprender la autonomía también en situación, conectando a la persona y el contexto a través de la acción.

La perspectiva transaccional o contextualizada corresponde al estudio de las relaciones cambiantes entre los aspectos psicológicos y ambientales de las unidades holísticas, y de este modo, la unidad de análisis la constituyen entidades holísticas tales como eventos que implican personas, procesos psicológicos y ambientes, y desde esta noción, el todo no se compone de elementos separados, sino que se trata de una confluencia de factores inseparables que dependen unos de otros para su definición y significado, y tiende a una filosofía pragmática y relativista para el estudio de los fenómenos (Altman \& Rogoff, 1987; Werner, Altman, \& Brown, 1992). Luego, el foco se encuentra en acciones que son inherentes a las relaciones entre actores y aspectos (personas, contexto, etc.) por sobre lo que toma lugar en cada uno de ellos por separado. De este modo, los diferentes actores y aspectos no se consideran entidades separadas (como alguna forma de interacción), sino que elementos que se coconstituyen a través de relaciones y cambian en el tiempo, enfatizando en la interconexión de los humanos con sus contextos y la contingencia de esa conexión que es inherente a las diferentes situaciones de la vida diaria (Cutchin \& Dickie, 2013; Dickie, Cutchin, \& Humphry, 2006), y de este modo, el acceso a la ocupación se busca a partir de situaciones de la vida diaria y no necesariamente a partir de actividades o desempeños.

De este modo, una racionalidad de autonomía transaccional o contextualizada incorporaría en un primer orden, la idea de la existencia de una 
entidad holística (en este caso autonomía) que se compone de factores inherentemente relacionados entre sí, y no enfatizará en alguno específico (como puede ser el factor individual) según se establece en la idea hegemónica de autonomía que ha sido previamente descrita.

Un elemento de aporte en la discusión sobre autonomía y participación, es la idea de agencia. Investigación previa ha sugerido que existe una relación entre agencia y participación (Borell, Asaba, Rosenberg, Schult, \& Townsend, 2006) y que existe la necesidad de conocer de mejor forma cómo las condiciones para la generación de agencia se dan cuando las personas se involucran en ocupaciones con otras personas. Si bien el concepto de agencia se describe tradicionalmente como la capacidad de una persona de actuar o llevar cabo según las diferentes situaciones que vive, la agencia es un concepto complejo que tiene diferentes bases teóricas y que también ha progresado en su racionalidad desde el énfasis individual hacia el relacional (Bandura, 2001) (Bandura, 2006).

Desde una perspectiva ocupacional, la agencia puede considerarse un concepto central debido a su relación con la ocupación humana. Sin embargo, los fundamentos teóricos en los que la terapia ocupacional y la ciencia de la ocupación se han construido asumen que las personas participan en ocupaciones como agentes autónomos, con la capacidad de autodeterminación, hacer elecciones sobre su vida, y actuar de acuerdo a lo que consideran como significativo, lo que implica una comprensión individual de la agencia, en las que las nociones de autodeterminación son centrales, negando la posibilidad y relevancia que tiene la interdependencia (Nyman, 2013). A partir de esto, surge una pregunta esencial hacia las prácticas actuales de Terapia Ocupacional para personas con demencia: ¿Cómo se valora la interdependencia? ¿Se visualiza como un objetivo, un facilitador o una barrera para el desempeño? La interdependencia en el marco de la ocupación y participación en situaciones diarias como ejes de agencia son también elementos de aporte hacia la configuración de una racionalidad de la autonomía.
A partir de lo anterior, desde una perspectiva de la ocupación, el transaccionalismo y la agencia, la autonomía puede visualizarse como una entidad que no necesariamente responde de forma única a la noción individual de competencia, sino que depende también del establecerse a partir de los otros, y de los componentes situacionales ajenos al individuo.

De este modo, la racionalidad frecuentemente categorial e individual de la terapia ocupacional en torno a la autonomía, puede ver una oportunidad de modificación hacia una idea de la autonomía desde el transaccionalismo, la interdependencia y la agencia, considerando la idea de posibilitar situaciones de la vida diaria para agencia de autonomía

por parte de las personas con demencia siendo partes de un contexto con los demás componentes relevantes de la vida cotidiana, como lo son las otras personas. $\mathrm{Al}$ respecto, el ambiente físico y social tiene un impacto facilitador o limitador en sus demandas hacia las personas con demencia (Lawton \& Nahemow, 1973). Uno de los principales componentes en relación con la satisfacción de necesidades de participación es la persona que cuida, usualmente un familiar, generándose un modelo triádico compuesto por la persona con demencia, el(la) cuidador(a) y el ambiente físico, que posiciona la relevancia de plantear intervenciones personalizadas y adaptadas a la realidad contextual de los sujetos (Algase et al., 1996) (Gitlin et al., 2008). Dichos elementos de contexto son esenciales de considerar en la idea de una racionalidad de la autonomía centrada en las situaciones, interdependencia y agencia.

\section{Premisas para un modelo transaccional o contextual de autonomía en la demencia}

De este modo, y bajo una mirada de la ocupación y participación, se propone el desarrollo de un modelo transaccional o contextual de autonomía en la demencia, incorporando los elementos conceptuales previamente desarrollados que incluyen la ocupación, la historia paradigmática de la demencia y su relación con la autonomía, la perspectiva 
transaccional, interdependencia y la agencia. Luego, un modelo transaccional de autonomía en la demencia puede incorporar las siguientes premisas: 1. La autonomía en la demencia es una entidad holística conformada, a lo menos, por los siguientes tres componentes o factores: (a) persona con demencia, (b)otras personas y entorno social, (c) ocupación y participación, los cuales son inseparables y determinantes de una única entidad denominada autonomía. La figura 1 esquematiza el modelo propuesto y sus componentes esenciales.

2. La autonomía es una situación o experiencia que posibilita agencia, por sobre una capacidad o un factor individual categorizable de manera descontextualizada.

3. La experiencia de autonomía se genera en contexto inherente entre la persona con demencia, las demás personas y el entorno social y las ocupaciones en las que se involucre (lo que supone un espacio y tiempo particular).

4. Los componentes presentan una interfaz que les comunica, cuyas características son particulares a cada situación cotidiana de autonomía, y por ende, podrían describirse y modificarse, lo que modificaría también la situación de autonomía puntual.

5. La evaluación de la situación de autonomía (ya sea en términos categóricos, de niveles, de percepción, u otro) puede darse únicamente si se considera el análisis de cada componente y de la interacción entre éstos.

Figura 1. Modelo transaccional o contextual de autonomía en la demencia

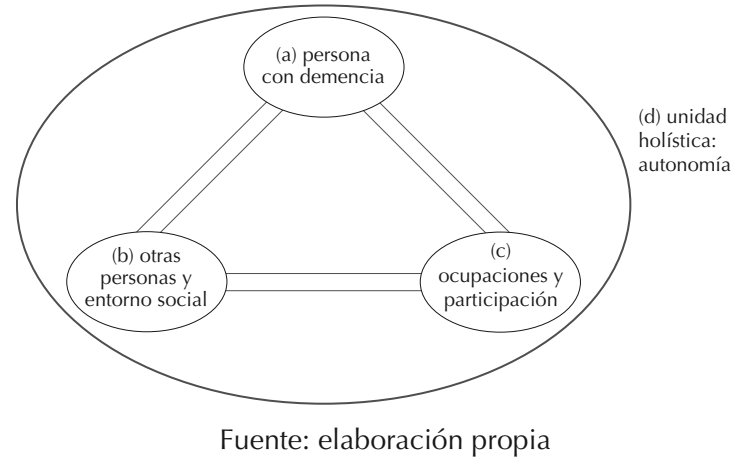

En el modelo, (a) representa a la persona con demencia que presentará características estables en términos de capacidades, siendo dicha estabilidad definible como tal en términos de un espacio y tiempo particulares; (b) representa el entorno social y las personas que forman parte de las situaciones de la vida diaria del sujeto; y finalmente (c) las ocupaciones en las que la persona y su entorno social desarrollen de manera conjunta.

\section{COnCLusiones}

La relación de la demencia con la autonomía representa un desafío teórico y práctico según los diferentes paradigmas a los cuales puede adscribirse. En su búsqueda de promoción de participación, autonomía y bienestar en las personas, y bajo la perspectiva de la ocupación, la Terapia Ocupacional y la Ciencia de la Ocupación encuentran pertinencia en la consideración de una racionalidad sobre la autonomía en la demencia que enfatice en lo relacional, el dinamismo y la interdependencia entre componentes individuales y contextuales, por sobre una visión categórica y centrada en la noción de capacidad individual. Una pregunta esencial ante esta aseveración es, ¿está efectivamente la Terapia Ocupacional y la Ciencia de la Ocupación posicionándose de esta forma en la práctica y el estudio de la autonomía y participación en personas con demencia? ¿Qué racionalidad sobre la autonomía en la demencia pudiese interpretarse a partir de las prácticas actuales de Terapia Ocupacional?

Es posible sintetizar elementos teóricos que permiten la construcción de nuevos modelos de racionalidad para autonomía en la demencia, que sustenten prácticas e investigación. Al respecto, este manuscrito realiza la propuesta de un modelo transaccional o contextualizado de la autonomía en la demencia, con miras a aportar con nuevas reflexiones conceptuales que sirvan a la práctica contemporánea y futura.

La discusión generada posiciona la necesidad de generar mayor investigación que logre enmarcarse de mejor forma en la interdependencia y la 
transacción por sobre la relevancia de las capacidades individuales y la idea de nteracción de componentes situacionales separados los unos de los otros, poniendo en tensión la idea de la ocupación como un desempeño individual que puede ser únicamente evaluado por medio de categorización y logro objetivo.

El modelo propuesto, autonomía como entidad holística, abre nuevas interrogantes a través del estudio de sus componentes, las que pueden aportar a comprender de mejor forma las experiencias de autonomía y la promoción de agencia cotidiana para dichas situaciones de la vida. Al respecto, interrogantes iniciales que emergen son, entre otras, ¿qué características de la persona con demencia posibilitan una mayor agencia para la autonomía? ¿Qué cualidades del ambiente y las otras personas promueven dicha agencia de mejor forma? ¿Cómo podría la Terapia Ocupacional evaluar la autonomía bajo un modelo transaccional o contextualizado y proponer intervenciones a partir de éste?

\section{REFERENCIAS BIBLIOGRÁFICAS}

Agich, G. (1994). Autonomy in Alzheimer disease. In E. Giacobini \& R. Becker (Eds.), Alzheimer disease: Therapeutic strategies (pp. 464-469). Oxford: Oxford University Press.

Algase, D. L., Beck, C., Kolanowski, A., Whall, A., Berent, S., Richards, K., \& Beattie, E. (1996). Need-driven dementia-compromised behavior: An alternative view of disruptive behavior. American Journal of Alzheimer's Disease and Other Dementias, 11(6), 10-19.

Altman, I., \& Rogoff, B. (1987). World views in psychology: Trait, interactional, organismic and transactional perspectives. In D. Stokols \& I. Altman (Eds.), Handbook of environmental psychology (Vol. 15, pp. 1-40). New York: Wiley.

Bandura, A. (2001). Social cognitive theory: an agentic perspective. Annual Review of Psychology, 52, 1-26. doi:10.1146/annurev. psych.52.1.1

Bandura, A. (2006). Toward a Psychology of Human Agency. Perspectives on Psychological Science, 1(2), 164-180. doi:10.1111/ j.1745-6916.2006.00011.x

Borell, L., Asaba, E., Rosenberg, L., Schult, M., \& Townsend, E. (2006). Exploring experiences of "participation" among individuals living with chronic pain. Scandinavian Journal of Occupational Therapy, 13(2), 76-85.

Brannelly, T. (2011). Sustaining citizenship: people with dementia and the phenomenon of social death. Nursing Ethics, 18(5), 66271. doi:10.1177/0969733011408049
Corporación Profesional Alzheimer y otras demencias. (2012). Propuesta de Plan Nacional de Alzheimer y otras Demencias. Retrieved from http://www.coprad.cl/coprad/wp-content/ uploads/2012/12/Resumen_ejecutivo.pdf

Cuijpers, Y., \& van Lente, H. (2014). Early diagnostics and Alzheimer's disease: Beyond "cure" and "care." Technological Forecasting and Social Change. doi:10.1016/j.techfore.2014.03.006

Cutchin, M., \& Dickie, V. (2013). Transactional Perspectives on Occupation. North Carolina: Springer. doi:10.1007/978-94-0074429-5

Dekkers, W. (2004). Autonomy and the lived body in cases of severe dementia. In R. Purtilo \& H. ten Have (Eds.), Ethical foundations of palliative care for Alzheimer's disease (pp. 115-130). Baltimore: Johns Hopkins University Press.

Departamento de Salud Pública Pontificia Universidad Católica de Chile. (2008). Informe final estudio de carga de enfermedad y carga atribuible. Retrieved from http://epi.minsal.cl/epi/html/invest/cargaenf2008/Informe final carga_Enf_2007.pdf

Diccionario Etimológico. (2014). Retrieved from http://etimologias. dechile.net/

Dickie, V., Cutchin, M., \& Humphry, R. (2006). Occupation as transactional experience: A critique of individualism in occupational science. Journal of Occupational Science, 13(1), 83-93.

Donoso, A. (2003). La enfermedad de Alzheimer. Revista Chilena de Neuro-Psiquiatría, 41(2), 13-22.

Dörr, O. (2005). Aspectos fenomenológicos y éticos del envejecimiento y la demencia. Revista Mé, (133), 113-120.

Fuentes, P., \& Slachevsky, A. (2005). Enfermedad de Alzheimer: Actualización en terapia farmacológica. Revista Médica de Chile, 224-230.

Garand, L., Lingler, J., Conner, K., \& Dew, M. A. (2009). Diagnostic Labels, Stigma, and Participation in Research Related to Demencia and Mild Cognitive Impairment. Research in Gerontological Nursing, 2(2), 112-121. doi:10.3928/19404921-20090401-04. Diagnostic

Gitlin, L. N., Winter, L., Burke, J., Chernett, N., Dennis, M. P., \& Hauck, W. W. (2008). Tailored activities to manage neuropsychiatric behaviors in persons with dementia and reduce caregiver burden: a randomized pilot study. The American Journal of Geriatric Psychiatry, 16(3), 229-39. doi:10.1097/JGP.0b013e318160da72

Hammell, K. (2004). Dimensions of meaning in the occupations of daily life. Canadian Journal of Occupational Therapy., 71(5), 296-305. Retrieved from http://www.ncbi.nlm.nih.gov/pub$\mathrm{med} / 15633880$

Hammell, K. (2009). Self-care, productivity, and leisure, or dimensions of occupational experience? Rethinking occupational "categories." Cana, 76(2), 107-114.

Innes, A., \& Manthorpe, J. (2013). Developing theoretical understandings of dementia and their application to dementia care policy in the UK. Dementia (London, England), 12(6), 682-96. doi:10.1177/1471301212442583

Instituto Nacional de Estadísticas. (2004). Chile : Proyecciones y estimaciones de Población. Total país 1950-2050. Retrieved from http://www.ine.cl/canales/chile_estadistico/demografia_y_vitales/proyecciones/Informes/Microsoft Word - InforP_T.pdf 
Instituto Nacional de Estadísticas. (2008). Población y Sociedad. Santiago. Retrieved from http://www.ine.cl/canales/chile_estadistico/demografia_y_vitales/demografia/pdf/poblacion_sociedad_enero09.pdf

Lawton, M. P., \& Nahemow, L. (1973). Ecology and the aging process. In C. Eisdorfer \& M. P. Lawton (Eds.), The psychology of adult development and aging (pp. 619-674). Washington, DC: American Psychological Association.

Marson, D. C. (2001). Loss of competency in Alzheimer's disease: conceptual and psychometric approaches. International Journal of Law and Psychiatry, 24, 267-283. doi:10.1016/S01602527(01)00064-4

Mitchell, G. J., Dupuis, S. L., \& Kontos, P. C. (2013). Dementia Discourse: From Imposed Suffering to Knowing Other-Wise. Journal of Applied Hermeneutics, 4.

Nyman, A. (2013). Togetherness in everyday occupations: How participation in on-going life with others enables change. Luleå tekniska universitet. Retrieved from https://pure. Itu.se/portal/sv/publications/togetherness-in-everydayoccupations(474e58b0-4e30-452d-b6ab-7e5bc6cd4109).html

Organización Mundial de la Salud. (2012). Dementia: A Public Health priority.

Pesonen, H.-M., Remes, A. M., \& Isola, A. (2013). Diagnosis of dementia as a turning point among Finnish families: A qualitative study. Nursing \& Health Sciences, (February). doi:10.1111/ nhs. 12059

Public Relations Institute of Australia Program Fight Dementia. (2011). Australia's Fight Dementia Action Plan. Retrieved from http://www.fightdementia.org.au/common/files/NAT/20111020_ Nat_Cam_ActionPlanBrief.pdf
Quiroga, P., Albala, C., \& Klaasen, G. (2004). Validación de un test de tamizaje para el diagnóstico de demencia asociada a edad, en Chile. Revista Médica de Chile, 132, 467-478.

Rigaux, N. (2011). Autonomie et démence: Pour une conception de l'autonomie "dementia-friendly." Gériatrie et Psychologie Neuropsychiatrie Du Vieillissement, 9(1), 107-15. doi:10.1684/ pnv.2010.0250

Robinson, A. L., Emden, C. G., Elder, J. a, Lea, E. J., Vickers, J. C., \& Turner, P. a. (2008). Multiple views reveal the complexity of dementia diagnosis. Australasian Journal on Ageing, 27(4), 183-8. doi:10.1111/j.1741-6612.2008.00316.x

Szot, J. (2003). La transición demográfico-epidemiológica en Chile, 1960-2001. Revista Española de Salud Pública, 77(5), 605-613.

Wadley, V. G., \& Haley, W. E. (2001). Diagnostic Attributions Versus Labeling: Impact of Alzheimer's Disease and Major Depression Diagnoses on Emotions, Beliefs, and Helping Intentions of Family Members. The Journals of Gerontology Series B: Psychological Sciences and Social Sciences, 56(4), P244-P252. doi:10.1093/ geronb/56.4.P244

Werner, C. M., Altman, I., \& Brown, B. B. (1992). A Transactional approach to interpersonal relations: Physical environment, social context and temporal qualities. Journal of Social and Personal Relationships, 9(2), 297-323. doi:10.1177/0265407592092008

Wilcock, a. a. (1998). Reflections on Doing, Being and Becoming. Canadian Journal of Occupational Therapy, 65(5), 248-256. doi:10.1177/000841749806500501

Zeilig, H. (2013). Dementia as a cultural metaphor. The Gerontologist, 1-10. doi:10.1093/geront/gns203 